\title{
Assessment of Vitamin BI2 status in patients with morbid obesity
}

\begin{abstract}
Vitamin B12 (VB12) is an essential water-soluble vitamin that also known as Cobalamin. The various forms of cobalamin include cyanocobalamin, hydroxycobalamin, methyl cobalamin and 5-deoxyadenosylcobalamin.It has vital functions of human body include; DNA synthesis, folate metabolism, optimal haemopoiesis, neurological function. Dietary deficiency of VB12 is rare, except in elderly people and long-term vegans. Nutritional deficiency, malabsorption syndromes, and gastrointestinal disorder are the most common causes of VB12 deficiency. Recently, studies documented that there is a deficiency of VB12 and folic acid in obese people compared with normal BMI controls. This review was aimed to evaluate VB12 level among patients with morbid obesity. The current data concluded that there is negative correlation between VB12 and body mass index. For that reason, VB12 must be routinely tested in morbidly obese people.
\end{abstract}

Volume 6 Issue 6 - 2017

\author{
Daniyah A AlKhawtani,' Mahmoud MA \\ Abulmeaty ${ }^{2}$ \\ 'Department of Clinical Dietitian, Prince Sultan Military Medical \\ City, Saudi Arabia \\ ${ }^{2}$ Department of community Health Sciences, King Saud \\ University, Saudi Arabia
}

Correspondence: Daniyah A AlKhawtani, Department of Clinical Dietitian, Prince Sultan Military Medical City, Riyadh, Saudi Arabia, Email dkhawtani@gmail.com

Received: June 06, 2017 | Published: June 19, 2017

Keywords: obesity, vitamin b12 deficiency

\section{Introduction}

Vitamin B12 (VB12) is an essential water-soluble vitamin that also known as Cobalamin. ${ }^{1}$ It is composed of a corrin ring consisting of four pyrrole units and a cobalt ion at the center of the ring. ${ }^{2}$ $1 \mathrm{VB} 12$ is manufactured by microorganisms present in the liver of animals bound to protein. The average B12 content of liver tissue is approximately $1.0 \mathrm{microgram} / \mathrm{g}$ of tissue in healthy adults. ${ }^{3}$ The various forms of cobalamin include cyanocobalamin, hydroxycobalamin, methyl cobalamin and 5-deoxyadenosylcobalamin, with the active forms of B12 in human metabolism being methyl cobalamin and 5-deoxyadenosylcobalamin.

In food, VB12 is complexed with protein. Food-bound B12 separates from proteins in the presence of stomach acid and pepsin. The released B12 combines with haptocorrins which are produced by the salivary glands and the gastric mucosa. Pancreatic proteases partially decompose the R proteins and releasing B12 to bind with an intrinsic factor in the small intestine. Usually the absorbance of VB12 is mediated by the intrinsic factor, which is a glycoprotein secreted by parietal cells of the stomach. The B12- intrinsic factor complex reaches the ileum part where it is absorbed. B12 requires almost 3 to 4 hours to enter the circulation. The total amount of VB12 stored in the body is about $2-5 \mathrm{mg}$ in adults. The liver takes up approximately 50 percent of the B12 and the remainder is transported to other tissues. If the circulating B12 exceeds the B12 binding capacity of the blood, the excess is lost in feces, skin and metabolic reactions. ${ }^{3}$

\section{Vitamin B I 2 vital functions}

DNA synthesis, folate metabolism, optimal haemopoiesis, neurological function. ${ }^{4}$ As well as, a cofactor for the enzymes methionine synthase and L-methylmalonyl-CoA mutase. Methionine synthase stimulates the conversion of homocysteine to methionine. Methionine is essential for the formation of S-adenosylmethionine which is a methyl donor for different substrates including DNA, RNA, hormones, proteins, and lipids. L-methylmalonyl-CoA mutase is required for transformation of L-methylmalonyl-CoA to succinyl$\mathrm{CoA}$ in the deterioration of propionate, an essential biochemical reaction in fat and protein metabolism and hemoglobin synthesis. ${ }^{3}$ VB12 is naturally founds in animal foods such as meat, fish, poultry, shellfish, and dairy product. In contrast, plant foods are considered poor sources of B12 but fortified foods such as breakfast cereals are readily available sources of VB12. ${ }^{5}$ Recommended dietary allowances (RDA) for VB12 are shown in Table 1.

Table I Recommended dietary allowances (RDAs) for Vitamin BI2 (per day)

\begin{tabular}{lllll}
\hline Age & Male & Female & Pregnancy & Lactation \\
\hline Birth to 6months & $0.4 \mathrm{mcg}$ & $0.4 \mathrm{mcg}$ & & \\
$7-12 \mathrm{months}$ & $0.5 \mathrm{mcg}$ & $0.5 \mathrm{mcg}$ & & \\
1-3years & $0.9 \mathrm{mcg}$ & $0.9 \mathrm{mcg}$ & & \\
$4-8$ years & $1.2 \mathrm{mcg}$ & $1.2 \mathrm{mcg}$ & & \\
9-13years & $1.8 \mathrm{mcg}$ & $1.8 \mathrm{mcg}$ & & \\
14+years & $2.4 \mathrm{mcg}$ & $2.4 \mathrm{mcg}$ & $2.6 \mathrm{mcg}$ & $2.8 \mathrm{mcg}$ \\
\hline
\end{tabular}

Dietary deficiency of VB12 is rare, except in elderly people and long-term vegans. Nutritional deficiency, malabsorption syndromes, and gastrointestinal disorder are the most common causes of VB12 deficiency. Twenty to fourty percent of elderly individuals suffer from Food-Bound Cobalamin Malabsorption (FBCM). Reduced gastric acid and/ or H. Pylori infection are most common causes of FBCM. In addition, long-term use of histamine blockers, proton pump inhibitors, metformin or gastric resection can adversely affect B12 absorption. ${ }^{6}$ B12 deficiency can leads to megaloblastic anemia, neurologic and psychiatric disorders, and in extreme cases pancytopenia (leukopenia, thrombocytopenia).

The diagnosis of VB12 deficiency is typically based on low serum VB12 levels, which are usually values less than 200pg/ $\mathrm{mL}(150 \mathrm{pmol} / \mathrm{L})$, along with clinical manifestation of the disease. Furthermore, measurements of methylmalonic acid and homocysteine have shown to be more accurate in the diagnosis of B12 deficiency than measurement of B12 levels alone. High levels of methylmalonic acid levels $(>0.4 \mathrm{micromol} / \mathrm{L})$ could be a more sensitive and reliable 
indicator of B12 status because they reflect a metabolic change that is highly specific to VB12 deficiency. On the other hand, elevated serum homocysteine levels $(>13$ micromol/L) might be poor indicators of B12 status because homocysteine levels affected by other factors like low vitamin B6 or folate levels. ${ }^{7}$

The pharmaceutical forms of VB12 supplements include oral, sublingual, nasal gel and injection. Cyanocobalamin is the compound that most readily converts to the active forms methylcobalamin and 5-deoxyadenosylcobalamin to be absorbed by the body. ${ }^{8}$ It is recommended that vegetarian and women planning to become pregnant take daily multivitamin supplements or add fortified cereals to their meals to ensure an adequate intake of 6 to $30 \mathrm{mcg}$ of vitamin B12 in a form that is readily absorbed. On the other hand, it is recommended that older adults should take from 100 to $400 \mathrm{mcg} /$ day of supplemental VB12.4 There is no toxic effects have been associated with large dose of VB12 from food or supplements in healthy people. ${ }^{4}$

Recently, studies documented that there is a deficiency of VB12 and folic acid in obese people compared with normal BMI controls. VB12 protects against superoxide injuries in endothelial cells while folic acid minimized oxidative stress. This review was aimed to evaluate VB12 level among people with obesity." "Obesity is a chronic, multifactorial disease involving excessive body fat with complex psychological, environmental (social and cultural), genetic, physiologic, metabolic and behavioral causes and consequences". ${ }^{10}$ Body mass index values are reliable indicator of obesity and are calculated from height (square meters) and weight (kilograms) measurements. ${ }^{11}$ In adults, a BMI between $30-40 \mathrm{~kg} / \mathrm{m}^{2}$ is known as obesity and above $40 \mathrm{~kg} / \mathrm{m}^{2}$ is considered morbid obesity. Furthermore, fat distribution measurements such as waist circumference and sagittal abdominal diameter are more sensitive indicator of health risks. ${ }^{12}$

There are several causes of obesity including a sedentary lifestyle, advancing age, low socioeconomic status and bad nutrition habits. ${ }^{11}$ Diabetes mellitus, coronary heart disease, insulin resistance, dyslipidemia, sleep apnea, hypertension, and atherosclerosis are the major disorders associated with obesity due to the production of excessive adipokines. Also, obesity contributes to immune dysfunction and is a major risk factor for certain cancers such as hepatocellular, esophageal, and colon cancer. ${ }^{13}$ Between 1990 and 2005 , the prevalence of obesity in adults increased from $20.8 \%$ to $36.2 \%$, with a $74 \%$ relative rise in just over a decade in the Kingdom of Saudi Arabia (KSA). In addition, the epidemic was significantly higher among women than men, ranging from $24.9 \%$ for females and $15.6 \%$ for males in 1989 , to $43.8 \%$ for females and $28.3 \%$ for males in 2005. Obesity is a key driver of cardiovascular diseases (CVD), diabetes, which account for $27 \%$ and over $20 \%$ respectively, in Saudi of adults (over 25years of age) Saudi population. ${ }^{14}$

Furthermore, in 2012, the Global Burden of Disease study found that elevated body mass index (BMI) was the major risk factor for morbidity in the KSA. There was study done to determine obesity prevalence and associated factors in the KSA, conducted a multistage of survey 10,735 persons aged 15years or older (51.1\% women), were interviewed. Sociodemographic data, health related habits and behaviors, diet, physical activity, chronic diseases, use of health care, and anthropometric measurements were collected through interviews. They found that $28.7 \%$ of Saudis were obese (BMI $\geq 30 \mathrm{~kg} /$ $\left.\mathrm{m}^{2}\right)$. The proportion of obesity was higher among women $(33.5 \%)$ and associated with marital status, education, history of chronic conditions, and hypertension. Obesity in men was associated with marital status, diet, physical activity, diagnoses of diabetes and hypercholesterolemia, and hypertension. ${ }^{15}$

\section{Literature review}

There was study done to investigate the relationship between VB12 and BMI along with insulin resistance (IR) in 116 middle aged obese and 103 aged matched healthy women. Weight, height, hip, waist circumferences, fasting and postprandial glucose, VB12 and folic acid (FA) levels, and lipid profiles were measured. Also, BMI and homeostasis model assessment estimated IR (HOMA-IR) was calculated. They found that VB12 level was low in obese patients and there is inverse relationship between B12 level and BMI, but not with HOMA-IR. ${ }^{16}$

In a cross-sectional dietary survey, 84 healthy volunteers ( 40 males and 44 females) from Jordan between the ages of 19 and 25years were randomly assigned to examine the relation between VB12 and body weight. Dietary intake was evaluated using food records from 3 consecutive days ( 2 weekdays and 1 weekend day) and serum B12 was measured for all subjects. They concluded that the prevalence of B12 deficiency among subjects was high, with concentration of B12 appears to increase as BMI increases. ${ }^{17}$

Another cross-sectional study examined the correlation of VB12 with obesity insulin resistance, metabolic syndrome in 976 patients (obesity: 414, overweight: 212, and control: 351). Anthropometric, blood pressure measurements and bioelectric impedance analysis (BIA) were recorded. Vitamin B12, folic acid, hemogram, insulin, ferritin, iron, total iron binding capacity and other biochemical tests were assayed. They concluded that low VB12 level was associated with obesity and overweight, but not with insulin resistance, metabolic syndrome and gender. VB12 was negatively correlated only with body mass index. ${ }^{18}$

In 2013, a retrospective randomized study done to evaluate the prevalence of VB12 deficiency in 65 morbidly obese patients (BMI>35). 32 subjects out of 65 were documented as vegetarian; 33 were documented as non-vegetarian. BMI, VB12 level was assessed and dietary information was collected. Average VB12 was 198.9pg/ $\mathrm{ml}$, which is below cut off levels. They found that 42 patients turned out to be B12 deficient, where as14 (22\%) were male and 28 (43\%) were female. Vegetarian dietary habits were found to be a substantial risk factor for B12 deficiency. VB12 deficiency more prevalent among obese population. ${ }^{19}$

Polycystic ovary syndrome (PCOS) described as abdominal obesity, insulin resistance, dyslipidemias and atherosclerosis. Kaya et al., ${ }^{20}$ investigated the association between VB12, folate and homocysteine level, homeostasis model and insulin resistance and obesity in 61 PCOS patients and compared with 61 matched controls. Homocysteine concentrations and homeostasis model index were higher, on the other hand VB12 level were lower in PCOS patients with insulin resistance compared with those without insulin resistance. Serum VB12concentrations were significantly lower in obese PCOS women in comparison with obese control women. They concluded that elevated homocysteine level, Insulin resistance and obesity were associated with lower serum VB12 concentrations in PCOS patients. ${ }^{20}$

Other study assessed serum albumin, calcium, phosphate, magnesium, ferritin, hemoglobin, zinc, folate, VB12, 25-OH vitamin D3, and intact parathormone (iPTH) in 232 morbidly obese subjects $\left(\mathrm{BMI} \geq 35 \mathrm{~kg} / \mathrm{m}^{2}\right)$ prior to bariatric surgery. In addition, copper, 
selenium, vitamin $\mathrm{B} 1, \mathrm{~B} 3, \mathrm{~B} 6, \mathrm{~A}$, and $\mathrm{E}$ levels were evaluated in a subsample which contain 89 subjects. They found that there were deficiencies in $12.5 \%$ of the subjects for albumin, $8.0 \%$ phosphate, $4.7 \%$ magnesium, $6.9 \%$ ferritin, $6.9 \%$ hemoglobin, $24.6 \%$ zinc, $3.4 \%$ folate, and $18.1 \%$ VB12. Furthermore, $25.4 \%$ severe deficiency of $25-\mathrm{OH}$ vitamin $\mathrm{D} 3$ associated with secondary hyperparathyroidism in $36.6 \%$ cases. Also they showed that $48.7 \%$ of the subjects had at least one of the most micronutrients deficiencies such as VB12, zinc and severe $25-\mathrm{OH}$ vitamin D3 deficiency. In the subsample, $32.6 \%$ showed there were deficiencies of selenium, $5.6 \%$ for vitamin B3, $2.2 \%$ for vitamin B6, and 2.2\% for vitamin E deficiency. Copper, vitamin B1, and vitamin A deficiency was found in none of the subjects. The result of the study indicated a high prevalence of micronutrient deficiencies in morbidly obese subjects. ${ }^{2}$

Furthermore, there was another study done to examine the association between folic acid and VB12 levels of overweight and obese women along with insulin resistance. They were involving 384 women with normal weight $(n=72)$ overweight $(n=125)$ and obese $(\mathrm{n}=187)$. Fasting blood glucose, fasting blood insulin, triglycerides, high-density lipoprotein cholesterol (HDL), low-density lipoprotein cholesterol (LDL)were assessed, BMI and HOMA-IR was computed. They found that there was no statistically significant correlation between VB12 levels of obese and overweight women with or without insulin resistance, whereas serum folic acid levels were significantly lower of the women with insulin resistance. Briefly, overweight and obesity in women are risk factors for VB12 deficiency. ${ }^{22}$

In 2016, there was a study developed to evaluate the relationship between obesity and serum Vitamin B12, FA, vitamin D (VD) concentrations in 100 obese adults at Umraniye Research Hospital. Serum VB12, FA and VD levels was measured after 8-12hours overnight fasting. In conclusion, the data indicated that there was significant correlation between VD and obesity but no correlation between vitamin B12 or FA levels and obesity. ${ }^{23}$

\section{Conclusion}

The data documented that there is negative correlation between VB12 and body mass index. For that reason, VB12 must be routinely tested in morbidly obese people.

\section{Acknowledgements}

None.

\section{Conflict of interest}

The author declares no conflict of interest.

\section{References}

1. Vitamin B12. Natural Medicines Comprehensive Database.

2. Vitamins: Water and Fat Soluble.

3. Dietary Reference Intakes for Calcium and Vitamin D.

4. Vitamin B12.
5. Da Silva L, Mc Cray S. Vitamin B 12: No One Should Be without It Practical gastroenterology. 2009;33(34):39-42.

6. Physicians. Cobalamin (vitamin B12) Deficiency, Investigation \& Management. UK: British Columbia medical association; 2013.

7. Robert $\mathrm{COH}$, Brown DL. Vitamin B12 deficiency. American Family Physician. 2003;83(12):1425-1430.

8. Vitamin B12. USA: National Institute of Health.

9. Baltaci D, Deler MH, Turker Y, et al. Evaluation of serum Vitamin B12 level and related nutritional status among apparently healthy obese female individuals. Nigerian Journal of Clinical Practice. 2017;20(1):99-105.

10. Health Care Guideline. Prevention and Management of Obesity for Adults. 3rd ed. USA: Institute for Clinical Systems Improvement; 2013.

11. Pi Sunyer FX. The obesity epidemic: pathophysiology and consequences of obesity. Obesity research. 2012;10(Suppl 2):97S-104S.

12. Gurevich Panigrahi T, Panigrahi S, Wiechec E, et al. Obesity: pathophysiology and clinical management. Curr Med Chem. 2009;16(4):506-521.

13. Redinger RN. The pathophysiology of obesity and its clinical manifestations. Gastroenterol Hepat. 2007;3(11):856-863.

14. Garawi F, Ploubidis GB, Devries K, et al. Do routinely measured risk factors for obesity explains the sex gap in its prevalence? Observations from Saudi Arabia. BMC public health. 2015;15(1):254

15. Memish ZA, El Bcheraoui C, Tuffaha M, et al. Obesity and associated factors-Kingdom of Saudi Arabia, 2013. Preventing chronic disease. 2013;11:140236

16. Baltaci D, Kutlucan A, Ozturk S, et al. Evaluation of vitamin B12 level in middle-aged obese women with metabolic and nonmetabolic syndrome: case-control study. Vitamin B12 level and obesity. 2012;42(5):802-809.

17. El-Qudah JM, Dababneh BF, Al-Qudah MM, et al. Serum Vitamin B12 levels related to weight status among healthy Jordanian students. Laboratory Medicine. 2013;44(1):34-39.

18. Baltaci D, Kutlucan A, Turker Y, et al. Association of vitamin B12 with obesity, overweight, insulin resistance and metabolic syndrome, and body fat composition; primary care-based study. Med Glas (Zenica) 2012;10(2):203-210.

19. Goyal R, Dhanuka S, Mehta V, et al. To Study The Vitamin B12 Status in Morbidly Obese Patients. International Journal of Food and Nutritional Sciences. 2013;2(2):73-76.

20. Kaya C, Cengiz SD, Satıroğlu H. Obesity and insulin resistance associated with lower plasma vitamin B 12 in PCOS. Reproductive biomedicine online. 2009;19(5):721-726.

21. Ernst B, Thurnheer M, Schmid SM, et al. Evidence for the necessity to systematically assess micronutrient status prior to bariatric surgery. Obes surg. 2009;19(1):66-73.

22. Kaner G, Adiguzel KT, Kurklu NS. Are Obese Women a Risk Group for Vitamin B12 and Folic Acid Deficiencies? Romanian Journal of Diabetes Nutrition and Metabolic Diseases. 2016;23(3):299-306.

23. Yagan FC, Olcum GG, Kurtulus D, et al. The relationship between obesity and serum Vitamin B12, folic acid, vitamin D concentrations in obese adults: a retrospective study. 2016 